\title{
La restructuration d'un combinat et ses répercussions sur le territoire local : L'acquisition de Dacia par Renault en Roumanie
}

The restructuring of a combine and its impacts on a local territory: The case of the acquisition of Dacia by Renault in Romania

Die Restrukturierung des Kombinats und ihre Auswirkungen auf das Lokalgebiet : Renaults Einkauf von Dacia in Rumänien

\section{Le Thiec Anaïs}

\section{OpenEdition}

Journals

Édition électronique

URL : http://journals.openedition.org/rge/3075

DOI : 10.4000/rge.3075

ISSN : $2108-6478$

Éditeur

Association des géographes de l'Est

Édition imprimée

Date de publication : 31 décembre 2010

ISSN : 0035-3213

\section{Référence électronique}

Le Thiec Anaïs, « La restructuration d'un combinat et ses répercussions sur le territoire local : L'acquisition de Dacia par Renault en Roumanie », Revue Géographique de l'Est [En ligne], vol. 50 / 3-4 | 2010, mis en ligne le 16 octobre 2011, consulté le 08 septembre 2020. URL : http:// journals.openedition.org/rge/3075; DOI : https://doi.org/10.4000/rge.3075

Ce document a été généré automatiquement le 8 septembre 2020

Tous droits réservés 


\title{
La restructuration d'un combinat et ses répercussions sur le territoire local : L'acquisition de Dacia par Renault en Roumanie
}

\author{
The restructuring of a combine and its impacts on a local territory: The case of \\ the acquisition of Dacia by Renault in Romania \\ Die Restrukturierung des Kombinats und ihre Auswirkungen auf das Lokalgebiet \\ : Renaults Einkauf von Dacia in Rumänien
}

Le Thiec Anaïs

\section{Introduction}

1 La Roumanie a connu de nombreux changements depuis les vingt ans qui nous séparent de la chute de Ceausescu. L'Etat-Parti n'existe plus et a peu à peu laissé place à un régime démocratique. La Constitution de 1991 a garanti la démocratie et la liberté ; la propriété privée est devenue un droit. Les citoyens roumains peuvent dès lors circuler librement et résider où ils le souhaitent. La Roumanie est entrée de plein pied dans le système financier et l'économie nationale s'est peu à peu intégrée au système mondial. Symboles de l'économie mondialisée, les firmes multinationales ont investi tardivement le marché roumain. C'est en 1996, suite à l'élection d'Emil Constantinescu, que la Roumanie a vu sa croissance économique décoller avec l'arrivée de nombreux investissements étrangers. Cette soudaine attractivité du pays pour les firmes multinationales est liée à sa promesse d'adhésion à l'Union Européenne. Avec vingt millions d'habitants, la Roumanie s'est imposée comme l'un des plus grands marchés potentiels d'Europe médiane pour les firmes multinationales. Cependant, force est de constater que seuls certains territoires sont attractifs pour les firmes multinationales. Plus encore, il apparaît que les investissements étrangers ne se répartissent pas de 
façon homogène sur le territoire. L'adaptationde l'économie roumaine aux marchés internationaux passe en partie par la reconversion des combinats, organes de base de l'industrie socialiste. Ils assuraient à la fois le contrôle des individus et de la collectivité, les services sociaux et la production de biens divers.

2 Cet article présente l'étude du cas particulier de la restructuration de Dacia par la firme multinationale Renault: quelles ont été les conséquences de la restructuration du combinat et comment ces changements se sont-ils traduits spatialement? Souvent douloureuses pour un territoire plus ou moins dépendant de l'activité industrielle locale, les conséquences de la restructuration sur le territoire local doivent être analysées. Si nous constatons une diminution de l'emploi qui est passé de trente mille salariés en 1998 à douze mille salariés en 2004, nous devrons comprendre comment l'arrivée de Renault a dynamisé et reconfiguré le « territoire Dacia ».

3 Aussi, nous étudierons dans quelle mesure l'arrivée de la firme a contribué au dynamisme économique local et expliquerons l'ancrage territorial de l'industrie automobile. Un récit rétrospectif de l'histoire régionale permettra de comprendre comment le Parti-Etat a créé l'ancrage territorial de l'industrie automobile autour de l'ancien combinat Dacia. Alors, nous pourrons appréhender les différentes étapes de la restructuration de Dacia par Renault et quelles en ont été les implications territoriales.

\section{I - Dynamisme économique en Arges et héritage territorial}

\section{A - Le territoire Dacia : une originalité économique en Roumanie}

4 La croissance économique roumaine est en grande partie le fruit des nombreux investissements des firmes multinationales depuis 1996. Bucarest, la capitale roumaine, et sa périphérie, l'Ilfov, ont absorbé $67 \%$ des investissements étrangers pendant la période allant de 1991 à 2008 ${ }^{1}$. Le premier judet ${ }^{2}$ de province, en termes d'investissements étrangers reçus pendant la même période est l'Arges avec six cent trente six millions d'euros. 93 \% de cette somme était destinée au secteur industriel. Les investissements émis en Arges sont à destination du secteur automobile. En effet, c'est dans la ville de Mioveni ${ }^{3}$, à une quinzaine de kilomètres au nord de Pitesti ${ }^{4}$ que la firme Renault a repris l'ancien combinat automobile Dacia. Depuis l'arrivée de Renault et de ses sous-traitants, le territoire est devenu très attractif. N'ayant pu imaginer le succès de la voiture à bas-coût, les sous-traitants regrettent aujourd'hui de n'avoir investi que pour de trop petites capacités de productions par rapport au rythme des lignes de production de Dacia. Alors qu'à la fin des années 1990, de nombreux terrains étaient disponibles à prix modéré, la pression foncière est telle qu'il est dorénavant difficile d'en acquérir. En ce qui concerne la population active, le salaire moyen tire vers le haut la moyenne des salaires roumains. En 2007, le salaire moyen en Arges était de 205 euros (221 euros pour les salariés de l'industrie), contre un salaire moyen de 194 euros pour l'ensemble de la Roumanie (188 euros pour les salariés de l'industrie) ${ }^{5}$. Les salariés de l'industrie dans le judet de l'Arges sont donc mieux payés que dans le reste de la Roumanie. Ces différents éléments en font, du point de vue économique, l'un des territoires les plus attractifs de Roumanie. Cependant, avant l'arrivée de Renault, rien ne laissait présager une telle attractivité de ce territoire. 
5 Le bassin d'emploi ${ }^{6}$ entourant Pitesti compte deux grandes industries : Dacia et la raffinerie Petrochim. La raffinerie ayant peu à peu vu son nombre de commandes baisser, les salariés sont régulièrement confrontés à des périodes de chômage technique. S'il y a en Arges une tradition de travail du bois, la prospérité économique de la région semble principalement due à la présence de Renault-Dacia. Alors qu'en 1998, le site de Dacia était dans une situation particulièrement inquiétante, le succès de sa restructuration a transformé l'ancien combinat en une usine-pilote qui exporte vers les différents sites d'assemblage de la Logan à travers le monde. Le territoire industriel en déclin a été transformé en une plate-forme de renommée internationale. Ainsi, «les choix de l'entreprise ont un fort impact, et établissent une relation allant dans le sens entreprise/ville. » (Fache J., 2008). L'industrie automobile, et plus particulièrement le constructeur Renault, est le moteur économique de son territoire environnant. Le judet de l'Arges se trouve alors dans une situation de dépendance économique vis-à-vis de l'industrie automobile. Avant que Renault ne rachète Dacia, la situation était similaire puisque sans repreneur, l'Arges aurait été une région industrielle sinistrée. Les liens étroits entre l'industrie automobile et le judet sont les conséquences de la place primordiale que prenaient les combinats à l'époque de la « construction socialiste ».

\section{B - Organisation du territoire : le combinat en héritage}

6 Le territoire roumain actuel est l'héritage de choix du passé. La Roumanie a entamé son développement industriel suite à son désengagement $\mathrm{du}^{\mathrm{CAEM}^{7}}$ et la construction industrielle s'est organisée sur les bases du modèle soviétique. L'objet de cet article étant la compréhension du "territoire Dacia ", un récit rétrospectif sur les étapes de l'industrialisation est nécessaire (Elissalde B., 2000)

7 L'usine Dacia n'a pas toujours construit des voitures, le développement industriel de Mioveni ${ }^{8}$ a commencé en 1936 avec une production industrielle pour l'armement. Après la seconde Guerre Mondiale, l'usine a été transformée pour produire des composantes mécaniques destinées aux usines d'assemblage de camions de Brasov. C'est en 1966 que les dirigeants roumains ont pris la décision de développer l'industrie automobile nationale, inexistante jusqu'alors, et de la situer à Colibasi, là où la main d'œuvre était déjà qualifiée dans la mécanique et où le réseau de fournisseurs était connu. Les dirigeants roumains ont dû se desserrer de «l'étau » soviétique pour diversifier leur industrie nationale. En 1956, l'armée soviétique s'est retirée du pays et la "désoviétisation» économique, sociale et culturelle a débuté en 1962. Nicolae Ceausescu, nommé au pouvoir en 1965, ainsi que les dirigeants du Congrès du Parti Communiste Roumain, ont refusé l'intégration non conditionnelle du CAEM pour laquelle la Roumanie avait un rôle de fournisseur de matières premières et de produits agro-alimentaires.Avec deux tiers de la population active qui travaillait dans l'agriculture avant 1965, la Roumanie a connu pendant les années de restructuration socialiste un rythme important de développement industriel. La planification a été entamée avec un vaste programme de développement de l'industrie lourde. L'industrie était considérée comme la seule voie viable de développement, celle de la modernité qui leur était refusée au sein du CAEM. La planification industrielle a alors eu un rôle structurant à l'échelle des judet et des villes. Le choix des dimensions des antennes de production était dépendant de critères d'économies d'échelle selon lesquels plus le volume de production est important, moindre est le coût d'une pièce. Il n'existait pas de seuil maximum ni d'économie de proportion. L'industrie s'est répartie sur le 
territoire sous la forme de combinats : établissements industriels de grande taille qui assuraient la fabrication des produits finis mais qui avaient également un rôle d'assistance sociale (enseignement, santé, culture, loisirs...) et de contrôle idéologique. Ce rôle de contrôle des idées distingue le combinat des usines paternalistes du XIX ${ }^{\circ}$ siècle. Dans l'organisation du combinat, aucune des ces trois attributions n'était supérieure à l'autre, il n'existait pas de hiérarchie (von Hirschhausen C., 1996).

8 Le Parti-Etat a décidé de développer trois combinats autour de Pitesti entre 1966 et 1968 : le combinat pétrochimique (Arpechim), le combinat de moteurs électriques et le combinat automobile (UAP). Les changements socio-économiques qui en ont découlé ont engendré une dynamique spatiale. Si le développement industriel de la région n'est pas uniquement lié à l'influence de Dacia, l'usine automobile a largement contribué aux changements dans la commune de Colibasi et dans la région environnante. La création de ces combinats se manifeste comme un choc violent qui s'accorde rarement avec le potentiel de développement de la ville; en découlent certains problèmes relevant de l'insuffisance des infrastructures, des transports, des services publics, et du manque de commerces (Groza, 1994). L'arrivée massive de populations dans ces «villes-usines» modifie en profondeur la structure de la population active. En nommant le combinat, UAP (usine automobile de Pitesti), il était clair que les dirigeants du Parti souhaitaient voir se développer la capitale du judet et non le village de Colibasi. En effet, Pitesti est l'une des villes de Roumanie qui a connu le plus fort accroissement de population depuis le recensement de 1948 puisqu'en cinquante ans, le nombre d'habitants a été multiplié par six. Cette augmentation de population pouvant être considérée comme artificielle puisque les populations étaient déplacées dans ces «villes-usines » pour y travailler. L'industrialisation a eu un rôle majeur dans la mise en place du contrôle territorial de la Roumanie.

9 Parallèlement à l'industrialisation, la «modernisation» du pays s'est également traduite par un changement des frontières administratives. En effet la réforme territoriale de 1968 a modifié le découpage administratif roumain en passant de 16 grandes régions à 39 judet. «La réforme administrative de 1968 avait trois buts: anéantir les anciens repères identitaires, créer d'autres repères territoriaux raccordés symboliquement à l'image du parti communiste, et créer des unités administratives qui devraient combiner localement le politique et l'économique» (O. Groza, 2001). L'échelle du judet devint alors l'échelle de base de la construction territoriale roumaine. Dans les travaux d'aménagement du territoire de l'époque, le Parti-Etat faisait en sorte que chaque judet développe une appartenance territoriale forte, le tout contrôlépar les organes locaux parti communiste. Ainsi, « pour répondre à son principe révolutionnaire d'inventer «l'homme nouveau ", elle "retourne» le territoire " (von Hischhausen B., 1997). Cette réforme avait pour but de gommer les repères identitaires liés au territoire. En créant l' « homme nouveau ", ils voulaient inscrire le pays dans l'ère du progrès. La création de ce "territoire nouveau» est alors passée par des investissements dans le secteur de l'industrie et la création des «villes-usines ». Les logiques de localisations industrielles de l'époque comprenaient la répartition équitable des productions sur l'ensemble du pays, une homogénéisation des judet et l'élimination des différences culturelles, économiques et sociales entre les villes et les villages. Afin de développer efficacement les judet, ces entités administratives ont du se spécialiser. Les industries devaient se trouver à proximité des matières premières nécessaires à la production et dans le voisinage d'un marché de consommation; le but étant d'assurer au judet une autosuffisance pour ce qui était de la consommation courante et de garantir des 
échanges entre les différents judet roumains pour les biens de consommation durables (réfrigérateurs, automobiles...).

Des villes petites ou moyennes, voire même des communes qui n'avaient pas le statut de ville ${ }^{9}$, ont accueilli ces combinats, ce qui a provoqué leur désorganisation : les villes se sont transformées en centres industriels avec l'arrivée de nouveaux travailleurs, et l'espace urbain a grossi de façon artificielle. Le potentiel de la ville était alors dépassé par les capacités industrielles créées et l'afflux de ces nouveaux travailleurs. Ainsi, la production automobile de l'UAP a commencé en 1968 et la commune de Colibasi s'est développée au rythme de l'usine. Alors que ce village rassemblait 5939 habitants en 1965, le recensement de 1992 en comptait 33 891. Parmi eux, 90\% logeaient dans les immeubles destinés aux ouvriers de Dacia, l'habitat traditionnel de ce sate (village) ayant été partiellement détruit pour construire ces immeubles.

\section{II - Le « processus de territorialisation - déterritorialisation - reterritorialisation » autour de l'industrie automobile en Arges}

\section{A - La construction d'un territoire autour de l'automobile}

11 A partir de 1968, le village de Colibasi s'est construit autour de l'usine Dacia. La figure 1 ci-après tente de montrer le "processus de territorialisation» lorsque l'Etat-central a décidé de créer le combinat. Le " processus de territorialisation-déterritorialisationreterritorialisation" (Raffestin C., 1986) nous permet de définir les différentes temporalités du territoire de l'industrie automobile. En effet, le processus de territorialisation est définie comme «(...) délimitation, centration et communication sont au coeur du processus de territorialisation et, par conséquent, de déterritorialisation et de reterritorialisation. ». Dans notre exemple, le combinat devient le nouveau lieu central: le lieu du contrôle et des prises les décisions. Le fonctionnement des "villes-usines », le réseau de communication, reposait sur ce combinat. Le regroupement de nombreux « membres » du combinat dans des immeubles à proximité immédiate de l'usine devait leur faciliter l'accès aux services et la proximité permettait également une surveillance de cette population. Les membres du combinat y étaient liés pour toutes leurs activités : l'éducation, les loisirs et même les biens alimentaires; aucune place n'était laissée à l'auto-organisation. "En bref, c'est un espace impersonnel, sans identité, dépourvu de signification (...)» (Groza, 1994). Les ouvriers et habitants des «villes-usines » ne choisissaient pas, ils recevaient ce qui leur était attribué : logement, alimentation ou activités culturelles. Lorsqu'un ménage souhaitait déménager, il devait faire une demande et attendre une autorisation. Le Parti-Etat se chargeait de définir les besoins de la population par voie législative comme par exemple, le programme d'alimentation rationnelle. Ainsi les individus subissaient le territoire : ils n'en étaient pas les acteurs. Les véritables acteurs de l'espace roumain étaient l'Etat-Parti et les combinats. 
Figue 1 : La territorialisation des « villes-usines »

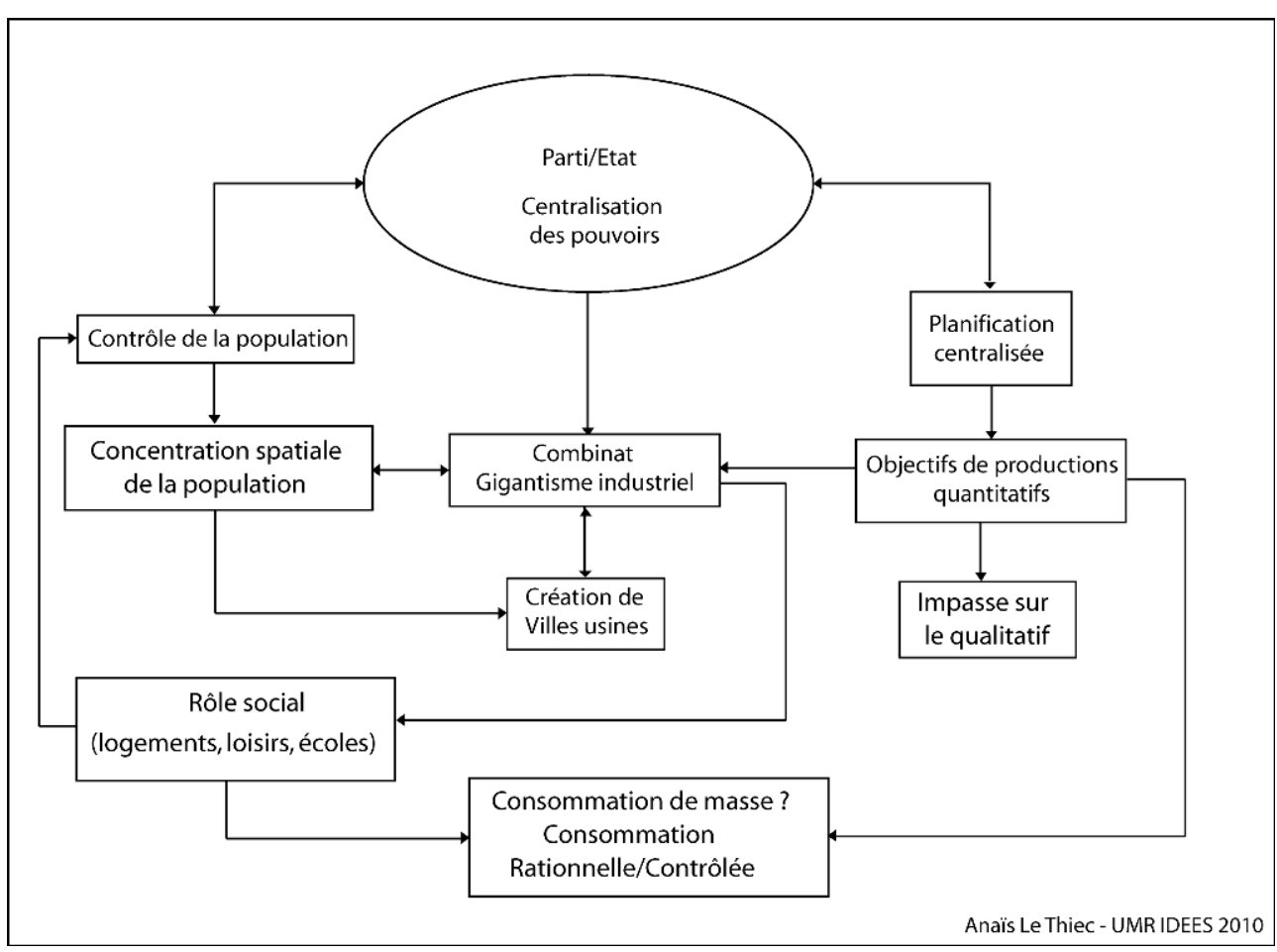

12 A la chute de Ceausescu, le système de fonctionnement des villes-usines s'est écroulé, le territoire s'est défait. Le rôle des combinats reposait sur trois piliers : la surveillance, le rôle social et la production de biens de consommation. La fin de l'Etat-Parti impliquait la fin du réseau de surveillance de la population. De même, le combinat ne pouvait plus fournir de services sociaux et ses "membres » sont devenus des salariés. Seuls deux éléments demeuraient du système passé : une commune (ville ou village) et un appareil productif. Après des années sans innovations majeures, les productions de ces usines étaient toutefois devenues obsolètes suite nouveaux biens de consommation disponibles suite à l'ouverture des frontières et à la concurrence internationale (Bafoil, 2004). Alors que pendant la période communiste les communes et les combinats fonctionnaient en interaction, le changement politique a supprimé les liens qui les unissaient auparavant. La chute de Ceausescu a entraîné une déterritorialisation ${ }^{10}$ de l'activité.

Après les dernières années de dictature, les roumains avaient des défiances à l'égard de l'Etat central et l'échelon local, peu habitué à la prise de décisions, a du reprendre sur ses épaules le poids de la gestion de la transition. Cette période floue a contribué au déclin de l'industrie par la perte de pouvoir et de contrôle des grands combinats, auparavant chargés de suivre le «Plan » et d'organiser la production socialiste. La loi $\mathrm{n}^{\circ} 15 / 1990$ a permis de réglementer les formes de réorganisation juridique des combinats qui devenaient alors des entreprises industrielles ou des sociétés commerciales.

Durant les premières années de changement politique, la perte d'emplois a été massive et les « industries géantes » ont bénéficié de subventions publiques pour survivre face aux risques sociaux que leur perte aurait engendrés. Dacia appartenait à cette catégorie d'industries protégées. "Avec la transition, il y a inversion des rôles : l'Etat sort de la sphère économique, domaine des entreprises, il entre dans la gestion du secteur social auparavant pris 
en charge par les entreprises » (Rey et ali, 2007). Les subventions publiques accordées à ces industries protégées ont provoqué un déséquilibre financier qui a amené la promulgation de la loi du 22 juin 1995 sur l'élaboration des procédures de liquidations et la fermeture des entreprises qui n'étaient pas privatisables (Popescu, 2000).

\section{B - Dacia ou le pari risqué de Renault}

En 1996, la politique de privatisation des entreprises nationales a été accélérée. Le projet de Renault de développer la voiture à 5000 euros a été choisi par le gouvernement roumain même s'il y avait de nombreux sceptiques à l'égard de ce projet et que l'usine était loin de correspondre aux standards de la production mondialisée. Cependant après l'échec de l'acquisition de Skoda au début des années quatre-vingt-dix, la firme multinationale souhaitait intégrer les marchés émergents des pays d'Europe Centrale et Orientale et l'acquisition de Dacia, même risquée, lui donnait cette opportunité.

\section{1 - Négociations entre la firme multinationale et l'Etat roumain}

16 L'acquisition de Dacia ne manquait pas d'avantages pour Renault. En effet, avec vingt millions d'habitants, la Roumanie est le second marché de consommation des PECO derrière la Pologne. La Roumanie et le site Dacia présentent des avantages compétitifs et comparatifs puisque la main d'œuvre roumaine est qualifiée et que les salaires restent faibles ${ }^{11}$ (Guran-Nica, 2004). En achetant Dacia, Renault reprenait un bassin d'emploi qualifié depuis trente ans dans la construction automobile, un savoir-faire ambiant propre à ce que Marshall décrivait dans les districts industriels. Un autre avantage non-négligeable pour la firme française est que les salariés roumains sont souvent francophones, ce qui facilite la communication. A l'échelle nationale, le « risque-pays » de la Roumanie pour les investisseurs étrangers a diminué au fur et à mesure des avancées des négociations d'adhésion à l'Union Européenne. Dacia était également pour le groupe Renault-Nissan l'achat d'une nouvelle marque, la marque nationale, qui faisait déjà $86 \%$ des ventes de voitures neuves sur le marché national pendant les années quatre-vingt-dix. L'obtention d'une nouvelle marque permet aux groupes automobiles d'être moins sensibles aux fluctuations du marché international et d'amortir les difficultés conjoncturelles (Archambaud O., Garcier R., 2001).

17 En reprenant un ancien combinat et un bassin d'emploi presque entièrement dépendant de la production automobile, la firme multinationale avait une position avantageuse lors des négociations pour la reprise du site. Dans un premier temps, Renault voulait qu'on lui laisse le temps de se positionner sur le marché local et de développer son concept de voiture à 5000 euros. La firme a alors négocié des accords fiscaux pour se protéger pendant quelques années de la concurrence étrangère. La firme a obtenu des exonérations fiscales pour les premières années de production, et des protections douanières. Afin que les consommateurs roumains achètent davantage de voitures produites en Roumanie, le gouvernement a interdit l'importation de véhicules d'occasion pendant cinq ans.

18 A l'échelle du bassin d'emploi, Renault ne voulait pas reprendre les trente-mille salariés et a donc négocié avec le gouvernement pour organiser un plan de licenciement massif. Ce dernier s'est déroulé en vingt étapes: quatre fois par an pendant cinq ans. Si les salariés décidaient de quitter l'entreprise, ils obtenaient des 
primes de licenciement proportionnelles à leur ancienneté. Nombreux ont été les salariés qui sont partis d'eux-mêmes, la moyenne des primes accordées étant de trois mille euros, soit plus de deux ans de salaires. Certains salariés en ont profité pour rejoindre leur région d'origine.

Comme il a été vu précédemment, la création de l'usine de production automobile avait créé une dynamique, un effet moteur sur Mioveni et sur toute la région de Pitesti. De la même façon, il faut s'interroger sur la perte d'emplois ou la reconversion professionnelle de plusieurs milliers de salariés sur le bassin d'emploi. Aller au delà du constat premier de la chute de l'emploi industriel dans le secteur automobile est nécessaire. En effet, pendant les dix ans de transition post-communiste, la production automobile était assez faible avec 470 voitures par jour et les voitures qui en sortaient étaient toujours les Dacia 1300, répliques de la Renault 12 des années soixante-dix. Les consommateurs roumains devaient attendre en moyenne un an et demi avant d'obtenir leur voiture. De plus, les fournisseurs historiques de Dacia, comme l'ancien combinat sidérurgique de Galati qui fournissait la tôle (sur les rives du Danube et de la Mer Noire), peinaient à fournir les composantes. Les performances de Dacia à l'extérieur des frontières roumaines étaient faibles avec seulement $3 \%$ des ventes à l'étranger et les salariés de l'usine Dacia n'étaient pas certains d'être rémunérés à la fin du mois. Renault a donc investi à un moment où Dacia ne pouvait plus continuer à produire seule et son arrivée a été considérée comme un "sauvetage", dernier espoir pour l'industrie automobile locale et nationale.

\section{2 - Changement de gouvernance au sein de l'usine et implications territoriales}

Renault est donc arrivé à Mioveni en 1999 et a changé le mode de gouvernance de l'entreprise pour l'adapter aux normes de productions occidentales. Avant que la firme ne rachète Dacia, l'usine était davantage gérée par des hommes politiques que par des professionnels de la production automobile. Le système de gouvernance était bureaucratique et ne pouvait pas s'adapter aux fluctuations du marché. Les responsables avaient des difficultés à dialoguer directement avec les salariés qui étaient répartis en équipe de quatre-vingt personnes, trop nombreux pour une communication efficace. Pendant la période communiste et les dix années qui ont suivi la chute du conducator, les ouvriers avaient pris l'habitude de travailler lentement afin d'éviter le chômage technique qui ne leur permettait d'obtenir que 60 \% de leur salaire. Ce système bureaucratique a été remplacé par un système triangulaire entre l'administration, les gestionnaires et les salariés. Après avoir mis en place de nouvelles lignes de productions, Renault a mis en place les UET (unités élémentaires de travail). Les UET sont «des groupes cohérents d'une vingtaine de personnes qui travaillent ensemble dans le cadre d'une même unité de temps et d'une même fonction opérationnelle. » Ces équipes sont responsables de leurs productions (qualité, coût, délais, ressources humaines) et cette responsabilisation des équipes a permis de faciliter le dialogue, d'accélérer la réactivité et a finalement amélioré la productivité. Une politique managériale nommée «monozokuri $»^{12}$, dépassant le cadre strict de la production industrielle au sein de l'usine, est aujourd'hui appliquée à Dacia. Renault cherche à maximiser son profit pour obtenir le plus de valeur ajoutée possible sur une voiture. Un des moyens pour les constructeurs d'être plus performant sur la construction de la voiture comme produit fini est de sous-traiter certaines pièces. Renault a donc repris Dacia, mais les sous- 
traitants habituels de Renault ont suivi le constructeur pour reprendre une partie de la production réalisée auparavant par l'entreprise roumaine.

La mise en place d'un réseau de sous-traitants par Renault a eu des répercussions sur le territoire national et local. La firme multinationale a transféré certaines opérations, autrefois réalisées par Dacia, vers des sous-traitants et équipementiers. ${ }^{13}$ La mise en place d'un réseau de sous-traitance est l'un des facteurs principaux de la réussite des firmes multinationales à l'étranger. Les équipementiers « traditionnels » de Renault ont suivi le constructeur français en Roumanie et se sont installés à proximité immédiate de l'usine dans la Zone Industrielle Fournisseurs. L'organisation à flux tendus de l'industrie automobile incite les sous-traitants à se regrouper autour du donneur d'ordre. Ce mode d'organisation à flux tendus, le toyotisme, est largement répandu dans l'industrie automobile mondiale. Il permet la synchronisation des productions et livraisons entre les sous-traitants et le constructeur automobile. Sur la plate-forme de Mioveni, la firme française envoie aux sous-traitants le film du montage des voitures cinq jours à l'avance, ce qui leur permet d'anticiper l'ordre dans lequel ils devront fournir les pièces sur la chaîne de montage (en fonction du modèle, de la couleur). Ce fonctionnement à flux tendus implique des délais de production de courte durée, et le constructeur n'a plus à supporter les coûts de stockage, alors répercutés sur les fournisseurs. A Mioveni la production automobile a produit un "système pyramidal » (Scott, 2001) puisque le donneur d'ordre, en haut de la chaîne de production, a attiré dans son sillage de nouvelles entreprises de sous-traitance, habituées à travailler avec le constructeur français dans la plupart des pays où il investit (Valeo, JCI). Outre les sous-traitants de premier rang qui totalisent plus de 6000 emplois dans le judet de l'Arges, nombreuses sont les entreprises dépendantes de la production automobile locale puisque Renault totalise soixante-treize sous-traitants de premier rang répartis dans toute la Roumanie (Bourdin et ali, 2009). De plus, le réseau de production et de distribution Dacia s'étend à travers le Monde par l'intermédiaire du groupe Renault

Ainsi, l'arrivée de Renault à Mioveni a permis une reterritorialisation ${ }^{14}$ de l'industrie automobile en Arges: le bassin d'emploi qui entoure l'usine automobile fait partie des plus dynamiques de Roumanie et le réseau Dacia s'étend désormais à travers le Monde.

\section{III - Polarisation autour de Pitesti et de Mioveni}

Afin de constater les changements spatiaux qui se sont opérés depuis l'arrivée de Dacia en Roumanie, il est nécessaire de comparer le territoire avant et après le rachat de Dacia. Les recensements de 1992 et de 2002 apportent un éclairage à l'échelle des communes. Le territoire étudié est délimité par les zones de migrations pendulaires des salariés de Dacia; il s'étend sur 56 kilomètres ${ }^{15}$ autour de Mioveni et sur trois judet, l'Arges, l'olt et la Dâmbovita (figure 2).

Après la chute de Ceausescu, la Roumanie a vu son taux de chômage augmenter rapidement. Nombreux étaient les salariés mi-paysans mi-ouvriers qui «faisaient la navette " quotidiennement pour aller travailler dans les combinats. Lors du changement politique de 1989, certains ont repris leurs terres pour redevenir paysans (von Hirschhausen, 1996). De la même façon, les jeunes diplômés qui étaient souvent envoyés vers un emploi loin de leur localité d'origine ont voulu retourner chez eux. La nouvelle liberté de circuler a permis aux citoyens roumains de déménager là où ils le souhaitaient. 
Pitesti et Mioveni ont alors été des villes attractives du fait de leur dynamisme économique. La population totale de Pitesti a continué à augmenter pour arriver à 187107 habitants en 2000 (soit $115 \%$ d'augmentation depuis 1990). A Colibasi ${ }^{16}$, qui était encore considéré comme un village en 1990, le nombre d'habitants a augmenté de $154 \%$ en onze ans pour arriver à 36357 en 2001. Les industries de l'Arges, comme Dacia, étaient "protégées " grâce aux subventions accordées par l'Etat, et ce judet perdait moins d'emplois que d'autres régions roumaines. Une tertiarisation de l'économie se mit alors en place et les personnes désirant créer leur entreprise (dans le commerce, le tourisme et les nouveaux services de type banque et assurances) choisissaient alors de s'installer dans cette région, moins chère que Bucarest, et cela a contribué à la diversification économique du territoire. L'attractivité économique locale étant dépendante du dynamisme des industries de base que sont la raffinerie Arpechim et Dacia, principalement.

Figure 2 : Le bassin d'emploi de Renault-Dacia (2010)

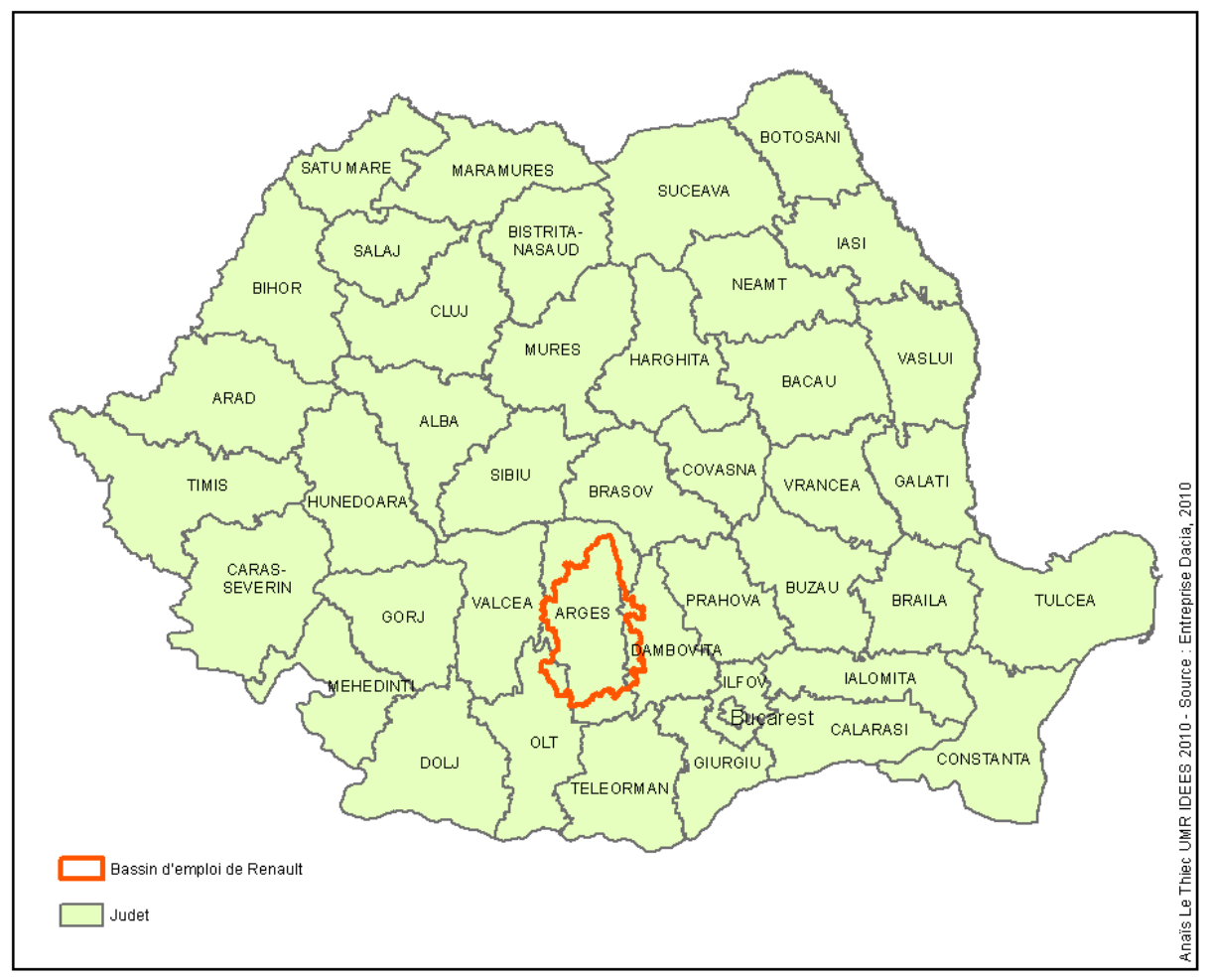


Figure 3 : Bassin d'emploi de Renault : les dynamiques industrielles

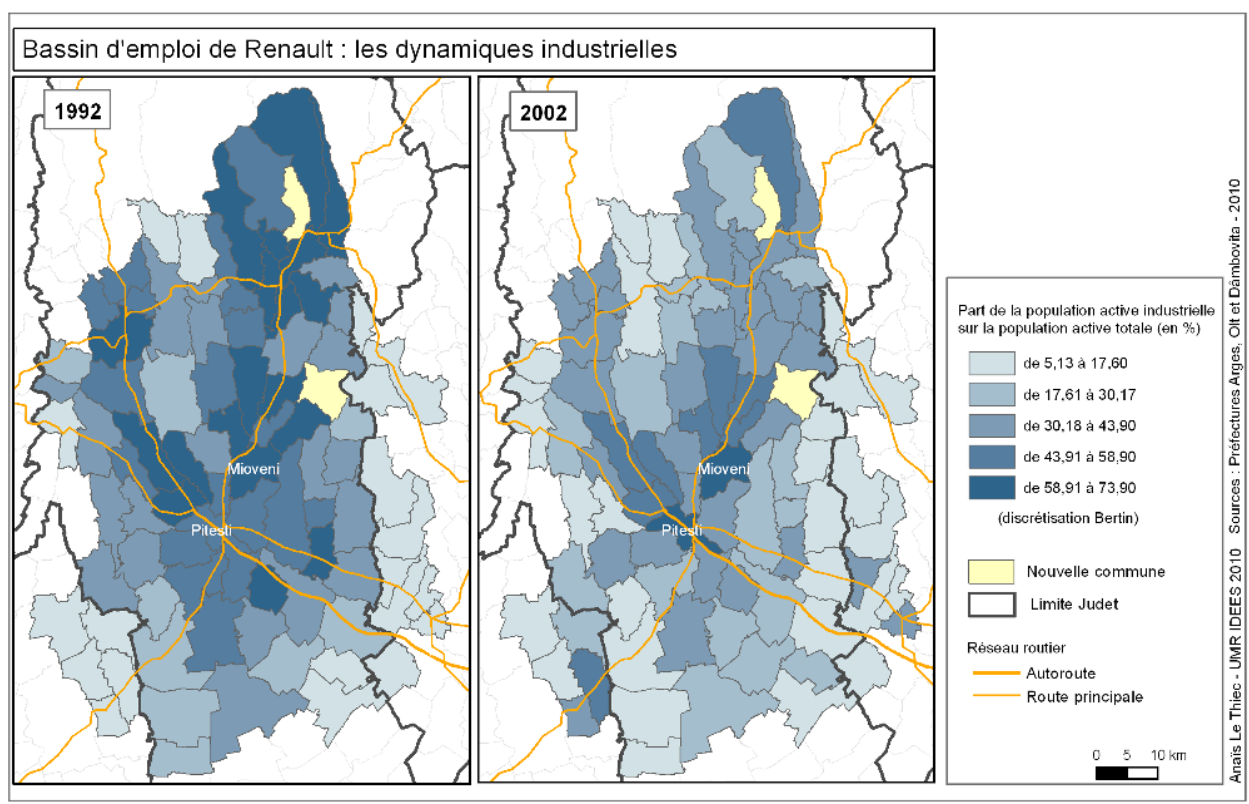

La croissance démographique de l'Arges a été interrompue en 2001, probablement suite à l'arrivée de Renault, au plan de licenciement et aux primes qui étaient proposés pour les départs volontaires. En 1992, 40\% de la population active occupée de l'Arges travaillait dans l'industrie contre $30 \%$ en 2008 et le nombre d'emplois est passé de 130000 à 76300 . Quant au taux de population active agricole, il est resté à $30 \%$ entre ces deux périodes. Plus précisément, les cartes ci-dessus (figure 3) montrent dans quelles communes le nombre d'habitants travaillant dans l'industrie a diminué. Alors que la population active industrielle de 1992 résidait davantage dans les communes rurales et faisait probablement la navette quotidiennement pour se rendre sur son lieu de travail, la carte de 2002 nous montre que la population active industrielle s'est resserrée dans les vallées urbanisées où passent les routes principales qui mènent à Brasov et Sibiu. Mais nous constatons aussi qu'elle se concentre principalement dans les villes de Mioveni et de Pitesti. Le schéma ci-après (figure 4) synthétise la dynamique territoriale du bassin d'emploi. 
Figure 4 : Dynamique industrielle du bassin d'emploi de Renault-Dacia

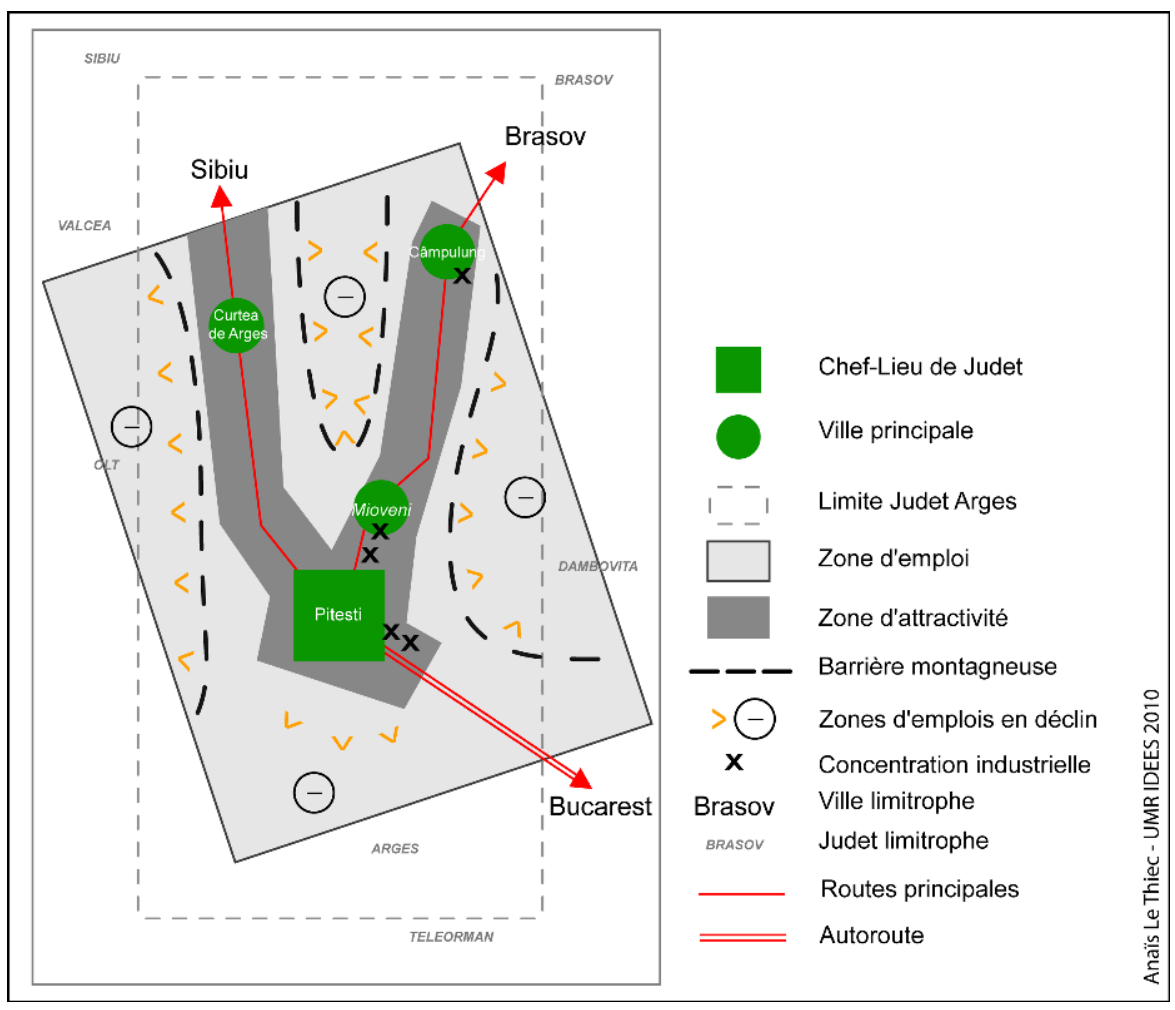

\section{Conclusion}

Moteur économique régional, la firme multinationale Renault a su créer une dynamique locale grâce au succès du projet de la voiture à $5000 €$. La mise en place d'un réseau de sous-traitants et la prospérité apparente des activités liées à la production automobile ont, par effet d'entraînement, produit une diversification des activités économiques. Depuis la création du combinat en 1968, la production automobile a été un élément majeur de la structuration du territoire en Arges. La politique de planification territoriale a entraîné l'évolution des villes de Pitesti et de Mioveni autour des combinats; le changement politique de 1989 a bouleversé les relations entre les combinats et les communes, et les individus ont pu redevenir des acteurs de l'espace. L'arrivée de Renault a ensuite redessiné le territoire. Nous avons pu constater une polarisation des activités dans des espaces précis, notamment autour de Mioveni et de Pitesti.

La production automobile est donc ancrée sur le territoire depuis plus de quarante ans et contribue depuis quelques années à la croissance économique de la Roumanie. Cependant, il reste des incertitudes quant à la prospérité de l'activité sur ce territoire. Les firmes multinationales ne sont pas forcément attachées sur le long terme aux territoires dans lesquels elles investissent. Bien que la firme Renault ait investi plusieurs millions d'euros dans la restructuration de l'ancien combinat, le choix de rester à Mioveni dépendra des avantages compétitifs et comparatifs que le site apportera au groupe Renault-Nissan.

16. 


\section{BIBLIOGRAPHIE}

Archambaud O. et Garcier R., 2001, Une géographie de l'automobile, Paris, P.U.F.318 p.

Bafoil F., 2004, «Quel outil de développement pour les économies est-européennes ?», Critique internationale, $\mathrm{n}^{\circ} 25$ - 2004, p. $123-138$.

Bourdin S., Le Thiec A., Elissalde B., 2009, «Le changement spatial dans l'industrie automobile en Europe centrale et orientale : entre effets d'héritage, cycles de vie et transition ", Géocarrefour, Vol. 84/3, p. $193-204$

Chanaron. J.L. et Lung.Y, 1995, l'économie de l'automobile, Paris, La découverte, 124 p.

Elissalde B, 2000, « Géographie, temps et changement spatial », L’Espace Géographique, Vol. 3-00, p. $224-236$

Emsellem K., 2001, Les petites villes dans le système de peuplement de la Roumanie, thèse de doctorat soutenue à l'Université Panthéon-Sorbonne, Paris, 520 p.

Fache J., 2008, «Quand l'entreprise recompose l'espace acentral », Géographie Economie Société 2008/4, Vol. 10, p. $469-492$

Groza O., 1994, « Pacșani, ville industrielle de Roumanie : années de transition », L’Espace Géographique, vol 4-94, p. 329 - 341

Groza 0.,2001,«Les échelles spatiales de la territorialité roumaine : essai géographique sur l'identification territoriale », New Europe College Yearbook, Vol. 8-2000-2001, p. 221 - 271

Guran-Nica L, 2002, InvestiŢiistrăine directe, Dezvoltareasistemului de aşezaridinRomânia, Bucureşti, Editura Tehnică S.A.,178 p.

Hischhausen Von B., 1997, Nouvelles campagnes roumaines : paradoxes d'un « retour » paysan, Paris, Belin, $239 \mathrm{p}$.

HirschhausenVon C., 1996, Du combinat socialiste à l'entreprise capitaliste, Paris, L'Harmattan, 257 p.

Popescu C., 2000, Industria României în secolul XX, București, Editura Oscar Print, 284 p.

Raffestin C., 1986, « Ecogenèse territoriale et territorialité », in F. Auriac et R. Brunet, Espaces, jeux et enjeux, Paris, Fayard, p. 175-185.

Rey V. et alii, 2007, Atlas de la Roumanie, Montpellier, La Documentation Française, 208 p.

Scott, A. J., 2001 Les régions et l'économie mondiale, Paris, L'Harmattan, 189 p.

http://www.insse.ro/cms/rw/pages/index.ro.do [dernière consultation le 31 octobre 2010]

Autres données recueillies en Roumanie dans le cadre de l'enquête de terrain (2010):

Les flux d'investissements étrangers Roumanie de 1991 à 2008, leur répartition par judet et par secteur d'activité.

Données sur la population recueillies dans les Préfectures de l'Arges, de l'olt et de la Dâmbovita.

\section{NOTES}

1. Office national du registre du commerce (Oficiul national al registrului comert). 
2. Les judet roumains sont l'équivalent des départements français, niveau NUTS 3.

3. Carte de localisation - figure 2.

4. Pitesti est la Préfecture de L'Arges.

5. Le salaire minimum roumain étant d'environ 125 euros.

6. Le bassin d'emploi de Dacia s'étend sur trois judet, il est calculé par rapport aux données fournies par l'entreprise Dacia (figure 3).

7. CAEM : Conseil d'Aide Economique Mutuel.

8. Colibasi a changé de nom pour s'appeler Mioveni en 1996, lorsque la commune a obtenu le statut de ville.

9. Pour la définition de la ville roumaine, se référer à la thèse de Karine Emsellem.

10. Ibid.

11. Le salaire mensuel moyen net était de 194 euros en 2007 selon l'institut national des statistiques.

12. Le monozokuri comprend 5 engagements : fournir des produits porteurs de valeur ajoutée, favoriser une culture d'entreprise saine et ouverte, contribuer au développement d'une société globale en tant que citoyen, le respect de l'environnement et répondre aux attentes des actionnaires.

13. Selon ACAROM (association des constructeurs automobiles de Roumanie), l'arrivée des soustraitants a créé environ trente-mille emplois en Roumanie et six-mille à proximité de l'usine Dacia, si on ne compte que les sous-traitants et équipementiers de rang un.

14. Ibid.

15. Données entreprise 2010.

\section{RÉSUMÉS}

Vingt ans après la chute du régime communiste et du conducator Nicolae Ceausescu, la Roumanie a largement entamé sa transition vers l'économie de marché. Suite à la promesse d'adhésion à l'Union Européenne, les firmes multinationales ont investi le marché local et la croissance économique roumaine a commencé sa phase de décollage en 1999. L'ancien combinat automobile Dacia se situe à proximité de Pitesti, une centaine de kilomètres au nord-ouest de Bucarest. Avec plus de trente-mille salariés, Dacia était l'usine motrice de l'économie locale et les dirigeants roumains ont du trouver un repreneur. La firme multinationale française Renault a alors repris le combinat automobile en 1998 et de nombreux bouleversements ont eu lieu. Nous proposons d'étudier les changements spatiaux sur le territoire local liés à la restructuration de l'ancien combinat par Renault.

Twenty years after the communism fall and Conducator Nicolae Ceausescu, Romania has largely started its transition towards a market economy. Following the promise of accession to the European Union, multinational companies have invested into the domestic market and the Romanian economic growth began its take off in 1999. Dacia is situated near Pitesti, about a hundred kilometers in the North West of Bucharest. With more than thirty thousands employees, Dacia factory was driving the local economy and Romanian leaders had to find a new purchaser. The French multinational company Renault then acquired the enterprise in 1998 and a lot of changes occurred. The special case of spatial changes in the local aera of the Dacia conglomerate bought by Renault will be studied here. 
Zwanzig Jahre nach dem Fall des Kommunismus und des ConducatorNicolae Ceausescu hat Rumänien den Übergang zur Marktwirtschaft schon angegangen. Nach dem Beitrittsversprechen zur EU haben die multinationalen Konzerne in den Inlandsmarkt investiert; seid dem ist der Rumänische Wirtschaft nur noch gestiegen. Der Konzern Dacia liegt in der Nähe von Pitesti, 100 $\mathrm{km}$ nord-westlich von Bukarest. Mit mehr als 30000 Angestellten war Dacia der führende Betrieb der lokalen Wirtschaft. Als in 1999 die Stadt den Konzern Dacia nicht mehr behandeln wollte, müssten die Entscheidungstreffern einen Übernehmer finden. Wir interessieren uns hiermit für räumlicheVeränderungenim Lokalgebiet die besonders nach der Übernahme von Dacia in 1998 statt gefunden haben.

\section{INDEX}

Mots-clés : investissements directs étrangers, Renault-Dacia, Roumanie, temporalité, territoire Keywords : foreign direct investment, Renault-Dacia, Romania, temporality, territory Schlüsselwörter : Ausländische Direktinvestitionen, Gebiet, Renault-Dacia, Rumänien, Zeitlichkeit

\section{AUTEUR}

\section{LE THIEC ANAÏS}

Doctorante en géographie, Université de Rouen, UMR IDEES 6266, Laboratoire MTG, UFR de lettres et sciences humaines, 76821 Mont Saint Aignan Cedex, anais.lethiec@gmail.com 